\title{
LATTICE DYNAMICS, THERMAL EXPANSION AND SPECIFIC HEAT OF A LENNARD-JONES SOLID IN THE QUASI-HARMONIC APPROXIMATION ${ }^{\dagger}$
}

F. W. DE WETTE and L. H. FOWLER

Department of Physics, University of Texas, Austin, Texas 78712, USA

and

B. R. A. NIJBOER

Instituut voor Theoretische Physica, Universiteit van Utrecht, Nederland

Received 19 February 1971

\section{Synopsis}

In this paper we present the results of an ab initio calculation of the thermal expansion, the specific heat and the mean-square amplitude of vibration of a LennardJones crystal, carried out in the quasi-harmonic approximation. The dynamical problem is solved for 110 values of the density, and the thermal expansion is obtained from a proper minimization of the Helmholtz free energy $F(T, a)(2 a$ is the lattice constant). Particular attention is given to the strong density dependence of the vibrational frequencies and the resultant effects on the thermodynamic quantities.

1. Introduction. We consider a monatomic face-centered cubic lattice, in which the atoms interact according to a Lennard-Jones $(\mathrm{L} J)$ pair potential of the usual form,

$$
\varphi(r)=4 \varepsilon\left[(\sigma / r)^{12}-(\sigma / r)^{6}\right],
$$

where $\varepsilon$ and $\sigma$ are potential constants and $r$ is the distance between the particles. The lattice parameter of the equilibrium lattice will be called $a$, and is defined such that $2 a$ is the edge of the cubic elementary cell $(a \sqrt{ } 2$ is the nearest-neighbour distance). We shall frequently use the dimensionless quantity $\sigma / a$ to indicate the lattice parameter or the density.

In an earlier report ${ }^{1}$ ) (published several years ago) we presented some preliminary results of lattice dynamical calculations for such a lattice, carried out in the quasi-harmonic approximation. The vibrational frequencies $\omega_{j}(\boldsymbol{q}, a)$ and polarization vectors $\boldsymbol{e}_{j}(\boldsymbol{q}, \boldsymbol{a})$ ( $j$ is the polarization index) were

† Supported in part by the U.S. Air Force Office of Scientified Research under Grant No. AFOSR 1257-67. 
calculated for a large number of wave vectors $\boldsymbol{q}$ inside the first Brillouin zone (B.Z.), the calculation being repeated for a whole range of values of $\sigma / a$, corresponding to the densities of the noble-gas crystals between $0 \mathrm{~K}$ and their melting points. It was found that for the interaction (1.1) the frequencies $\omega_{j}(\boldsymbol{q}, a)$ depend strongly on $a$; more specifically, when $\sigma / a$ was varied between 1.29 and 1.25 , roughly corresponding to the density range of argon between $0 \mathrm{~K}$ and its melting point at $84 \mathrm{~K}$, the frequencies decreased by about $26 \%$.

In continuation of our earlier work, in the present paper we give the results of some ab initio calculations of the thermal expansion, the specific heat (as particular cases of the more general thermal and caloric equations of state), and the mean-square amplitude of vibration of a Lennard-Jones crystal in the quasi-harmonic approximation. From the frequencies $\omega_{j}(\boldsymbol{q}, a)$ one can calculate the Helmholtz free energy $F(T, v)$ as a function of temperature $T$ and volume per particle $v$, or as a function of $T$ and $a$. By minimizing the free energy for a given value of $T$ with respect to the lattice parameter $a$, one obtains the equilibrium lattice parameter $a(T)$ as a function of temperature, i.e. the lattice constant [or volume per particle $v(T)$ ] for zero pressure as a function of $T\left[p=-(\partial F / \partial V)_{T}\right]$. In a similar fashion one can calculate the entropy $S(T, v)$ and for example the specific heat $C_{V}(T, v)$ as certain integrals over the frequency spectrum, computed at the appropriate densities.

The principal aim of this work has been to carry out a rather complete model calculation of certain dynamical and thermodynamic properties of crystals, avoiding a number of approximations, such as restrictions to the low- and high-temperature limits, which have often been made in discussions of this kind. The only assumption that we have made is the validity of the quasi-harmonic approximation. In recent years a number of treatments of anharmonic crystals have been developed; in both the so-called perturbation theory ${ }^{2}$ ) and the self-consistent phonon approximation ${ }^{3}$ ) the effects of anharmonicity can be taken into account in an approximate way. However, as far as we know, until now no complete calculation has been carried out in either the quasi-harmonic or one of the anharmonic approximations, in which the dynamical problem has been solved for the entire range of densities. We want to stress that this is in principle the only correct way in which thermodynamic quantities like the free energy can be obtained as functions of volume as well as temperature.

As a model pair potential we have chosen the LJ potential (1.1) because of its analytical simplicity and the fact that for suitable choice of the parameters $\varepsilon$ and $\sigma$ it represents the interaction between noble-gas atoms reasonably well. Although our main goal has been to calculate thermal and caloric properties of a crystal for the pair interaction given by (1.1) rather than to reproduce theoretically the observed properties of real crystals, we 
shall, for the sake of illustration, compare our results with some experimental data obtained for the noble-gas crystals. Deviations between theoretical and experimental results will partly be due to the restriction to the quasi-harmonic approximation, partly to the inadequacy of the LJ potential (and the neglect of many-body interactions), and it is not always obvious which of these is the most important cause of the deviations. For a comprehensive comparison of theoretical and experimental properties of noble-gas crystals we refer to the literature, e.g. the review articles by Pollack ${ }^{4}$ ) and Horton ${ }^{5}$.

In sec. 2 we discuss the solution of the dynamical equations and present some of the results for the dispersion curves and the frequency spectrum. In sec. 3 we describe the calculation of the thermal expansion; as an illustration the results for $\mathrm{Ar}$ are compared with experiment. The calculations for the specific heat and the mean-square amplitude of vibration are given in sec. 4.

2. The dynamical problem. In the quasi-harmonic approximation the equations of motion of the atoms are given by

$$
M \ddot{u}_{\alpha}(\boldsymbol{l})=-\sum_{\boldsymbol{l}^{\prime}}^{\prime} \sum_{\beta} \varphi_{\alpha \beta}\left(\boldsymbol{l} \boldsymbol{l}^{\prime}\right) u_{\beta}\left(\boldsymbol{l} \boldsymbol{l}^{\prime}\right) .
$$

Here $M$ is the atomic mass, $u_{\alpha}(\boldsymbol{l})$ the $\alpha$ component of the deviation of the atom $\boldsymbol{l}$ from its equilibrium lattice position $\boldsymbol{r}^{l}(\alpha, \beta=x, y, z)$. $\sum_{l^{\prime}}^{\prime}$ indicates a summation over the lattice sites with $\boldsymbol{l}^{\prime}=\boldsymbol{l}$ excluded. Further,

$$
u_{\beta}\left(\boldsymbol{l l}^{\prime}\right)=u_{\beta}(\boldsymbol{l})-u_{\beta}\left(\boldsymbol{l}^{\prime}\right),
$$

and

$$
\varphi_{\alpha \beta}\left(\boldsymbol{l} \boldsymbol{l}^{\prime}\right)=\frac{\partial^{2}}{\partial \alpha \partial \beta}[\varphi(\boldsymbol{r})]_{\boldsymbol{r}=\boldsymbol{r}^{\prime \boldsymbol{l}^{\prime}}}
$$

where $\boldsymbol{r}^{\boldsymbol{l}^{\prime}}=\boldsymbol{r}^{\boldsymbol{l}}-\boldsymbol{r}^{\boldsymbol{l}^{\prime}}$

The normal mode solutions of eq. (2.1) are of the form

$$
u_{\alpha}(\boldsymbol{l})=e_{\alpha}(\boldsymbol{q}) \exp \left[2 \pi \mathbf{i} \boldsymbol{q} \cdot \boldsymbol{r}^{\boldsymbol{t}}-\mathrm{i} \omega(\boldsymbol{q}) t\right],
$$

where the wave vector $\boldsymbol{q}$ lies within the 1 st B.Z. and is chosen in such a way that periodic boundary conditions are satisfied. The vibrational frequencies $\omega(\boldsymbol{q})$ and polarization vectors $\boldsymbol{e}(\boldsymbol{q})$ satisfy the equation

$$
\omega^{2}(\boldsymbol{q}) e_{\alpha}(\boldsymbol{q})=\sum_{\beta} D_{\alpha \beta}(\boldsymbol{q}) e_{\beta}(\boldsymbol{q}),
$$

where the dynamical matrix $D_{\alpha \beta}(\boldsymbol{q})$ is given by

$$
D_{\alpha \beta}(\boldsymbol{q})=(1 / M) \sum_{\boldsymbol{l}}^{\prime} \varphi_{\alpha \beta}(\boldsymbol{l})\left[1-\exp 2 \pi \mathrm{i} \boldsymbol{q} \cdot \boldsymbol{r}^{l}\right] .
$$


Here $\Sigma_{l}^{\prime}$ is a summation over the lattice with $l=0$ omitted. Performing the differentiations in (2.6) we obtain

$$
\begin{aligned}
& D_{\alpha \beta}(\boldsymbol{q})=\frac{1}{M} \sum_{\boldsymbol{l}}^{\prime}\left[\frac{\boldsymbol{r}_{\alpha}^{l} \boldsymbol{r}_{\beta}^{l}}{\left(\boldsymbol{r}^{l}\right)^{2}}\left\{\varphi^{\prime \prime}\left(\boldsymbol{r}^{l}\right)-\frac{1}{r^{l}} \varphi^{\prime}\left(\boldsymbol{r}^{l}\right)\right\} \frac{\delta_{\alpha \beta}}{r^{l}} \varphi^{\prime}\left(\boldsymbol{r}^{l}\right)\right] \\
& \times\left[1-\exp \left(2 \pi \mathrm{i} \boldsymbol{q} \cdot \boldsymbol{r}^{\boldsymbol{l}}\right)\right] \text {. }
\end{aligned}
$$

Substituting the $\mathrm{LJ}$ potential (1.1) one finds

$$
\begin{aligned}
& D_{\alpha \beta}(\boldsymbol{q})=\left(24 \varepsilon / M \sigma^{2}\right)(\sigma / a)^{8}\left[-28(\sigma / a)^{6} S_{16}^{\alpha \beta}(\boldsymbol{q})+8 S_{10}^{\alpha \beta}(\boldsymbol{q})\right. \\
& \left.\quad+\delta_{\alpha \beta}\left\{\frac{\mathbf{2} \boldsymbol{2}}{3}(\sigma / a)^{6} S_{14}(0)-\frac{5}{3} S_{8}(0)+2(\sigma / a)^{6} S_{14}(\boldsymbol{q})-S_{8}(\boldsymbol{q})\right\}\right],
\end{aligned}
$$

where the lattice sums are defined by

$$
\begin{aligned}
& S_{n}^{\alpha \beta}(\boldsymbol{q})=\sum_{\boldsymbol{l}}^{\prime} \rho_{\alpha}^{\boldsymbol{l}} \rho_{\beta}^{\boldsymbol{l}} \exp \left(2 \pi \mathrm{i} \boldsymbol{q} \cdot \boldsymbol{r}^{\boldsymbol{l}}\right) /\left(\boldsymbol{\rho}^{\boldsymbol{l}}\right)^{n}, \\
& S_{n}(\boldsymbol{q})=\sum_{\boldsymbol{l}}^{\prime} \exp \left(2 \pi \mathrm{i} \boldsymbol{q} \cdot \boldsymbol{r}^{\boldsymbol{l}}\right) /\left(\boldsymbol{\rho}^{\boldsymbol{l}}\right)^{n},
\end{aligned}
$$

and $\boldsymbol{\rho}^{\boldsymbol{I}}=\boldsymbol{r}^{\boldsymbol{l}} / a$. The linear homogeneous equations (2.5) have a solution only if

$$
\operatorname{det}\left|D_{\alpha \beta}(\boldsymbol{q})-\delta_{\alpha \beta} \omega^{2}(\boldsymbol{q})\right|=0 .
$$

This determinantal condition leads for every allowed wave vector $\boldsymbol{q}$ to three values for the frequencies $\omega(\boldsymbol{q})$ which are characterized by the polarization

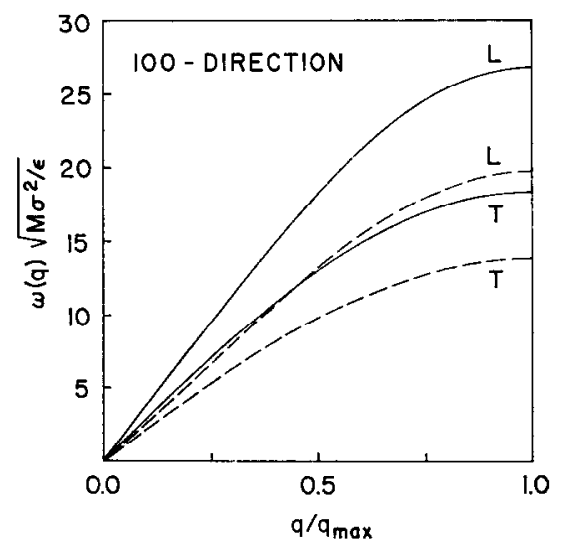

Fig. 1

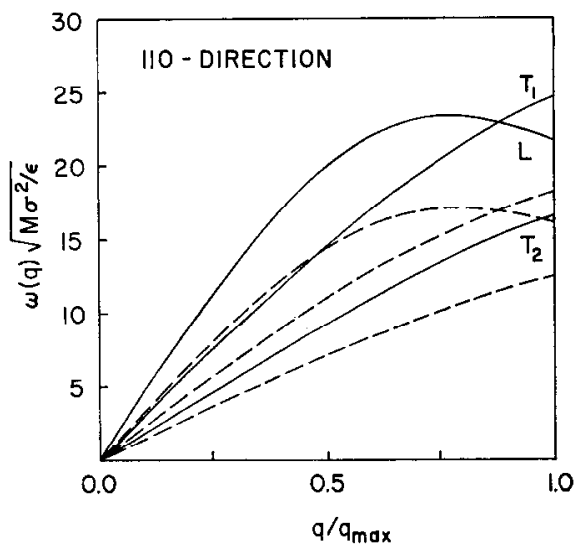

Fig. 2

Fig. 1. Phonon dispersion curves in the (100) direction for an fcc Lennard-Jones solid, for densities corresponding to $\sigma / a=1.29$ (solid curves) and 1.25 (dashed curves). $\mathrm{L}$ indicates the longitudinal branch, $\mathrm{T}$ the transverse branches.

Fig. 2. Phonon dispersion curves in the (110) direction for an fcc Lennard-Jones solid for densities corresponding to $\sigma / a=1.29$ (solid curves) and 1.25 (dashed curves). 
index $j(j=1,2,3)$. On account of the cubic symmetry of the lattice the wave vectors $q$ need only be chosen in the irreducible $1 / 48$ th part of the first B.Z.

The dynamical matrix $D_{\alpha \beta}(\boldsymbol{q})$ depends (except for the factor $\varepsilon / M \sigma^{2}$ ) essentially on $\sigma / \boldsymbol{a}$ and $\boldsymbol{q}$. Solutions of the eigenvalue problem (2.5) were obtained for a mesh of $10569 q$ vectors distributed uniformly in $1 / 48$ th of the B.Z. for each of 110 values of the parameter $\sigma / a$. The latter ratio was varied from $\sigma / a=1.200$ to $\sigma / a=1.309$ in steps of 0.001 . This range of densities covers the stable crystals of $\mathrm{Ne}, \mathrm{Ar}, \mathrm{Kr}$ and $\mathrm{Xe}$ from $\mathrm{OK}$ to the triple point temperatures $\left.{ }^{6}\right)$. In the numerical evaluation of the lattice sums a number of neighbours was taken into account, sufficient to obtain an accuracy of 1 part in $10^{5}$ in the sums. All calculations were performed on the CDC 6600 computer of the University of Texas at Austin.

Some of the results are presented in figs. 1-4. In fig. 1 we have plotted the dispersion curves $\omega_{j}(\boldsymbol{q})$ in the (100) direction for $\sigma / a=1.29$ and $\sigma / a=1.25$. The dispersion curves in the (110) direction for the same densities are given in fig. 2 . These $\sigma / a$ values correspond roughly to the case of solid $\mathrm{Ar}$ at $0 \mathrm{~K}$ and its melting point at $84 \mathrm{~K}$, repectively. The strong dependence of the frequencics on the density of the crystal is very striking indeed. For the longitudinal (L) branch in the (100) direction the ratio of the two frequencies for equal $q / q_{\mathrm{max}}$, for the two densities indicated above, varies between 1.7 and 1.6 for increasing $\sigma / a$; for the transverse (T) branch this is roughly 1.55 .

These results can be easily checked for small values of $\boldsymbol{q}$. In that case one can expand the elements of the dynamical matrix in powers of $q_{x}, q_{y}$ and $q_{z}$. One finds in lowest order

$$
\begin{aligned}
& D_{x x}(\boldsymbol{q})=\left(16 \pi^{2} / M\right)\left[q_{x}^{2}(A+C)+\left(q_{y}^{2}+q_{z}^{2}\right)(B+C)\right], \\
& D_{x y}(\boldsymbol{q})=\left(32 \pi^{2} / M\right) q_{x} q_{y} B .
\end{aligned}
$$

The other matrix elements follow from cyclic permutation of $x, y$ and $z$. Here

$$
\begin{aligned}
& A=\sum_{l}^{\prime}\left(x^{l}\right)^{4} \psi^{\prime \prime}\left[\left(r^{l}\right)^{2}\right], \\
& B=\sum_{l}^{\prime}\left(x^{l} y^{l}\right)^{2} \psi^{\prime \prime}\left[\left(r^{l}\right)^{2}\right], \\
& C=\frac{1}{2} \sum_{l}^{\prime}\left(x^{l}\right)^{2} \psi^{\prime}\left[\left(\gamma^{l}\right)^{2}\right],
\end{aligned}
$$

where $\psi\left(r^{2}\right)=\varphi(r)$. For the LJ potential we find

$$
\begin{aligned}
& A=168 \varepsilon(\sigma / a)^{12} S_{16}^{x x x x}(0)-48 \varepsilon(\sigma / a)^{6} S_{10}^{x x x x}(0), \\
& B=168 \varepsilon(\sigma / a)^{12} S_{16}^{x x y y}(0)-48 \varepsilon(\sigma / a)^{6} S_{10}^{x x y y}(0), \\
& C=-12 \varepsilon(\sigma / a)^{12} S_{14}^{x x}(0)+6 \varepsilon(\sigma / a)^{6} S_{8}^{x x}(0),
\end{aligned}
$$

where the definition of $S_{n}^{x x x x}$ and $S_{n}^{x x y u}$ is a direct cxtcnsion of the definitions 


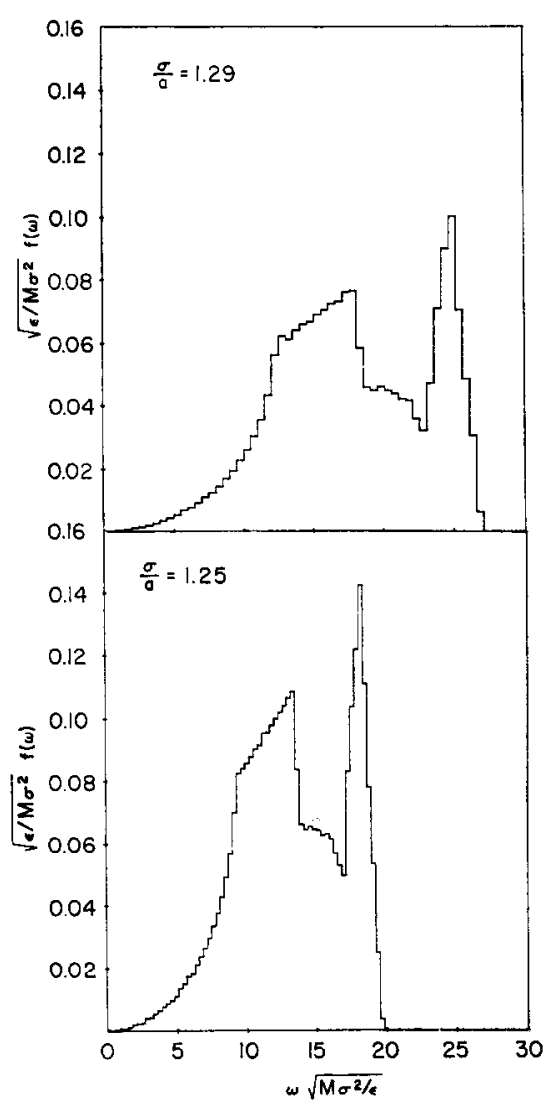

Fig. 3

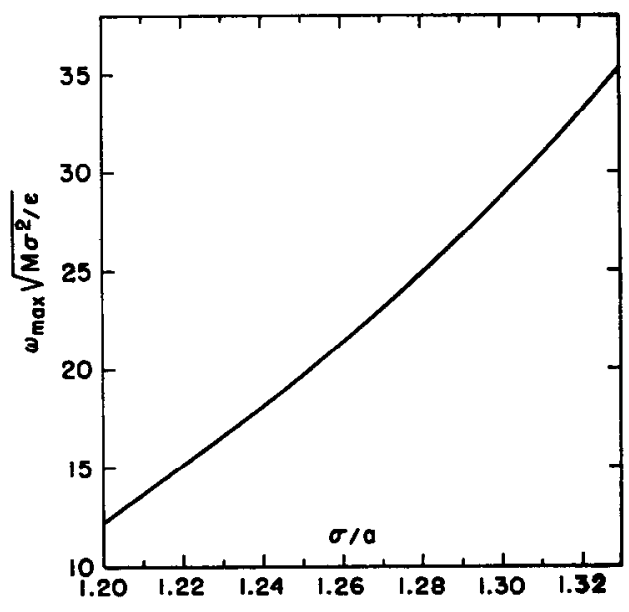

Fig. 4

Fig. 3. Frequency distributions for an fcc Lennard-Jones solid for densities corresponding to $\sigma / a=1.29$ and 1.25 .

Fig. 4. Maximum frequency for an fcc Lennard-Jones solid as a function of density for the density 1 ange corresponding to $\sigma / a=1.20-1.33$.

(2.9). For cubic lattices the lattice sums in (2.13) have been tabulated e.g. by Misra ${ }^{7}$ ). It is evident that the dynamical matrix, through $A, B$ and $C$, is strongly dependent on the value of $\sigma / a$. Furthermore it can be found quite easily from the expressions (2.11) that the slopes at the origin of the longitudinal and transverse dispersion curves in the (100) direction are proportional to $(A+C)^{\frac{1}{3}}$ and $(B+C)^{\frac{1}{2}}$ respectively. With this information ${ }^{\dagger}$ the slopes at $q=0$ of the dispersion curves in fig. 1 can be checked quite easily. At the same time this demonstrates the strong density dependence

+ We quote from ref. $7: S_{16}^{x x x x}(0)=0.03185 ; S_{10}^{x x x x}=0.3204 ; S_{16}^{x x y y}=0.01567$; $S_{10}^{x x_{y y}}=0.1409 ; S_{14}^{x x}(0)=0.06319 ; S_{\mathbf{8}}^{x x}(0)=0.6022$. 
of the vibrational frequencies (at least for small $q$ ) without having to solve the dynamical problem.

In fig. 3 we have plotted the frequency distributions for the same values of $\sigma / a$. We note that the relatively sharp peak at the high-frequency end of the spectrum is almost exclusively due to the "longitudinal" $\dagger$ frequencies whereas the broad peak more to the left in the spectrum is duc to the "transverse" modes. Again the large change in the frequency spectrum which results from a density change corresponding to a linear expansion of less than $4 \%$ is quite striking.

This is illustrated a little more explicitly in fig. 4 where the maximum frequency is plotted as a function of $\sigma / a$ for the range of $\sigma / a$ values considered here. In elementary discussions of the equation of state of solids one often makes use of the assumption that for all vibrational modes the quantity

$$
\gamma=-\mathrm{d}(\ln \omega) / \mathrm{d}(\ln V)
$$

is a constant (the so-called Grüneisen constant), independent of volume $V$ and the same for all modes ${ }^{8}$ ). We are now in a position to check this assumption. One can write for (2.14)

$$
\gamma=\frac{1}{3} \frac{\sigma / a}{\omega} \frac{\partial \omega}{\partial(\sigma / a)} .
$$

Restricting ourselves to $\omega=\omega_{\max }$ one may easily verify from fig. 4 that over the range of densities considered here $\gamma$ varies roughly from 4.9 (for $\sigma / a=1.20$ ) to 2.9 (for $\sigma / a=1.31$ ). So $\gamma$ turns out to be strongly dependent on the density; on the other hand it has nearly the same value for all modes.

3. Thermal expansion. As explained in the introduction, in order to find the thermal expansion, i.e. the lattice parameter as a function of temperature $a(T)$, one first has to evaluate the Helmholtz free energy $F(T, v)$ as a function of temperature $T$ and volume per particle $v$, or also as a function of $T$ and $a$.

In the quasi-harmonic approximation $F(T, a)$ has the form

$$
F(T, a)=\frac{1}{2} N \sum_{l}^{\prime} \varphi\left(\boldsymbol{r}^{\boldsymbol{l}}\right)+\frac{1}{\beta} \sum_{\boldsymbol{q}, j} \ln \left[2 \sinh \left\{\frac{1}{2} \beta \hbar \omega_{j}(\boldsymbol{q}, a)\right\}\right],
$$

where $N$ is the number of atoms, $\beta=1 / k_{\mathrm{B}} T$ and $\Sigma_{q}$ is a summation over the

† One should remember that for arbitrary directions of the wave vector $\boldsymbol{q}$ the vibrational modes are not strictly longitudinal or transverse. However, we have here defined the "longitudinal" frequency for given $\boldsymbol{q}$ as the frequency of that mode of which the polarization vector has the largest projection along $\boldsymbol{q}$; the other two frequencies for the same $\boldsymbol{q}$ belong to the "transverse" modes. 
$N$ values of $\boldsymbol{q}$ uniformly distributed throughout the first B.Z. The first term is the static lattice energy, and the second term is the contribution from the harmonic vibrations (including the zero-point energy). On substitution of (1.1) we have

$$
\begin{gathered}
F(T, a)=2 N \varepsilon\left[(\sigma / a)^{12} S_{12}(0)-(\sigma / a)^{\mathbf{6}} S_{\mathbf{6}}(0)\right] \\
+\frac{1}{\beta} \sum_{\boldsymbol{q}, j} \ln \left[2 \sinh \left\{\frac{1}{2} \beta \hbar \omega_{j}(\boldsymbol{q}, a)\right\}\right] .
\end{gathered}
$$

The lattice sums may be found in Misra ${ }^{7}$ ):

$$
S_{12}(0)=0.18956, S_{6}(0)=1.806740 \text {. }
$$

The function $F(T, a)$ can now be evaluated, for given values of $\sigma, \varepsilon$ and $T$, and for the whole range of $a$ values ( $\sigma / a$ values) by using the results for the vibrational frequencies discussed before. By minimizing $F(T, a)$ for given $T$ with respect to $a$, i.e. by determining $a$ from $\partial F(T, a) / \partial a=0$ we find the equilibrium lattice parameter $a$ for that temperature. For given $\varepsilon$ and $\sigma$ this procedure was carried out for a set of 20 values of $T$.

By way of example we show in fig. 5 the function $2 a(T)$ for the following

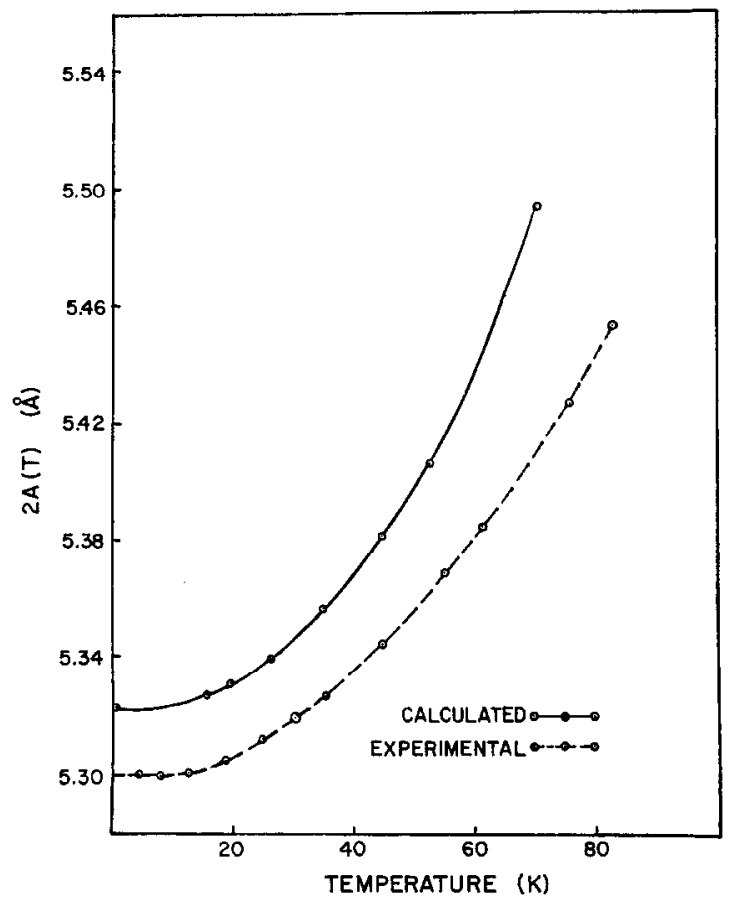

Fig. 5. Thermal expansion of an fcc Lennard-Jones solid. Solid curve: calculated lattice constant $2 a(T)$ for the choice of potential parameters $\varepsilon$ and $\sigma$ given in the text. Dashed curve: experimental thermal expansion curve for $\mathrm{Ar}^{12}$ ). 
choice of the potential parameters: $\varepsilon=164.3 \times 10^{-16} \mathrm{erg}, \sigma=3.409 \times 10^{-8}$ $\mathrm{cm}$. These are the values appropriate for Ar as given by Horton and Leech ${ }^{9}$ ) for a $(6,12)$ Lennard-Jones potential. The experimental values $\left.{ }^{10}\right)$ of $2 a(T)$ for $\mathrm{Ar}$ are shown in the same figure. One notices a slight discrepancy $(0.4 \%)$ between the experimental value $2 a(T=0)=5.300 \AA$ and the value $5.322 \AA$ obtained from the minimization procedure. Of course we might have obtained a complete fit between theoretical and experimental values of the lattice constant at $T=0 \mathrm{~K}$ by a minor adjustment of the potential parameters, but since we present these results merely as an illustration of the method rather than as a detailed calculation for argon we have not bothered to do so.

If the calculated curve in fig. 5 is shifted downwards to coincide with the experimental curve at $0 \mathrm{~K}$, we find that the quasi-harmonic approximation [using the Lennard-Jones interaction $(1,1)$ ] reproduces the lattice parameter within $0.2 \%$ up to half the melting temperature $\left.{ }^{11}\right)$. Hence the coefficient of thermal expansion is fairly well reproduced up to that temperature. At higher temperatures the deviations increase rapidly with temperature. It has been made plausible by recent approximate calculations of Leech and Reissland ${ }^{11}$ ) that the agreement at higher temperatures is substantially improved when anharmonic corrections are taken into account.

The thermal expansion results for $\mathrm{Kr}$ and $\mathrm{Xe}$ (that is, when potential parameters appropriate for $\mathrm{Kr}$ and $\mathrm{Xc}$ are used) are not qualitatively different from those of Ar discussed above and will not be presented here.

4. Specific heat. The specific heat at constant volume $C_{V}$ is, like all thermodynamic quantities, a function of two state variables, e.g. $C_{V}(T, v)$ or $C_{V}(T, p)$. However, in experimental or numerical presentations of this function it is usually implied that the second variable is held constant. For instance, if $C_{V}$ is derived from measurements of $C_{p}$ on a crystal under its own vapour pressure, one has essentially $p=0$. On the other hand, in numerical calculations of $C_{V}$ it is customary to use the vibrational spectrum at a fixed density, so that $v=$ constant; usually the volume at absolute zero $v_{0}$ is chosen. Sometimes the experimental results are presented in this way, but of course the reduction from measured $C_{p}\left(T, p_{\text {vapour }}\right)$ to $C_{V}\left(T, v_{0}\right)$ contains uncertainties as long as the dependence of the vibrational spectrum on $v$ is not accurately known.

The function $C_{V}(T, v)$ is given by

$$
C_{V}(T, v)=k_{\mathbf{B}} \sum_{\boldsymbol{q}, j} \frac{\left[\beta \hbar \omega_{j}(\boldsymbol{q}, a)\right]^{2} \exp \left[-\beta \hbar \omega_{j}(\boldsymbol{q}, a)\right]}{\left\{1-\exp \left[-\beta \hbar \omega_{j}(\boldsymbol{q}, a)\right]\right\}^{2}},
$$

where $a$ is the lattice parameter corresponding to $v$. With (4.1) one can in principle evaluate the complete function $C_{V}(T, v)$, e.g. $C_{V}(T, p=0)$ or $C_{V}(T, v=$ const. $)$. 
In evaluating the sum over $\boldsymbol{q}$ in (4.1) at low temperatures one encounters a peculiar difficulty, caused by the discreteness of the $\boldsymbol{q}$ mesh. Since the mesh is finite there exists a smallest frequency, $\omega_{\min }$ in the calculated spectrum. For temperatures smaller than $\hbar \omega_{\min } / k_{\mathrm{B}}$ the specific heat as calculated with (4.1) becomes anomalously small as a result of the exponential in the numerator. In order to obtain $C_{V}$ at the lowest temperatures (which lie well below $\hbar \omega_{\min } / k_{\mathrm{B}}$ ) a special procedure has to be followed. Since the original $q$ mesh of 10569 points in the irreducible part of the B.Z. is too coarse around the origin for the evaluation of $C_{V}$ at low temperatures, we have used two $q$ meshes of 10569 points each ; one covering the entire B.Z. and one covering the inner $\left(\frac{1}{27}\right)$ th of the zone (linear dimensions $\frac{1}{3}$ of the coarse mesh). Since even this procedure is not accurate enough for very low temperatures we have used a special procedure, based on the Debye approximation, in the region of reciprocal space immediately around the origin, where the Debye approximation is strictly valid. This method has been described in detail elsewhere ${ }^{12}$ ).

In fig. 6 we present curves for $C_{V}(T, v)$ as functions of $T$ for two fixed values of $v$, namely $v(0 \mathrm{~K})$ and $v(40 \mathrm{~K})$ as they follow from the calculated

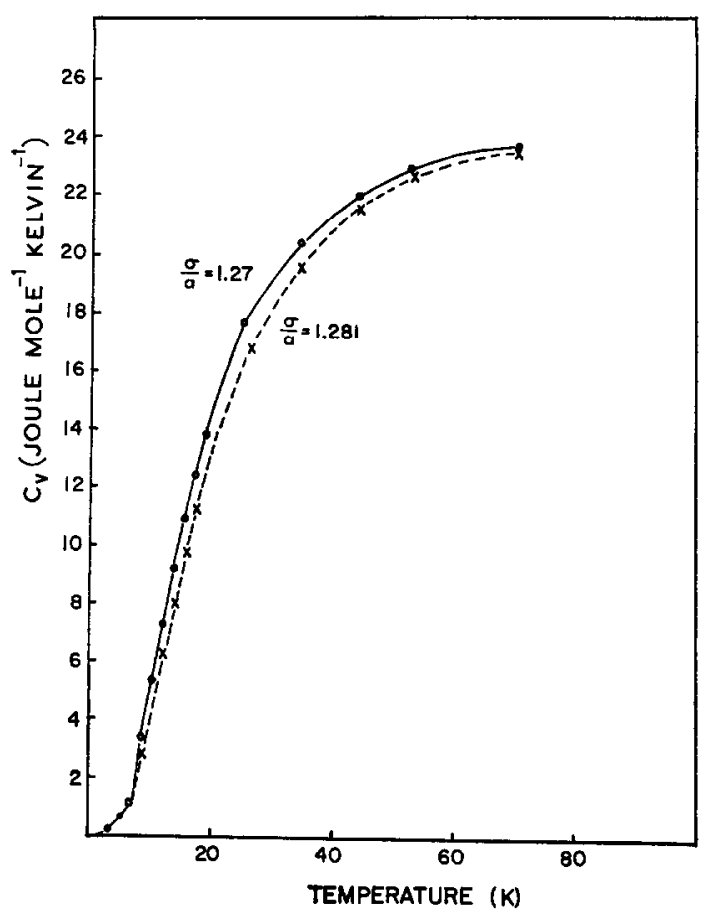

Fig. 6. Specific heat $C_{V}(T, v)$ for an fcc Lennard-Jones solid as a function of temperature with volume $v$ held constant. Dashed curve: $v=$ volume at $0 \mathrm{~K}$ and $p=0 \mathrm{~atm}$; solid curve: $v=$ volume at $40 \mathrm{~K}$ and $p=0 \mathrm{~atm}$. 


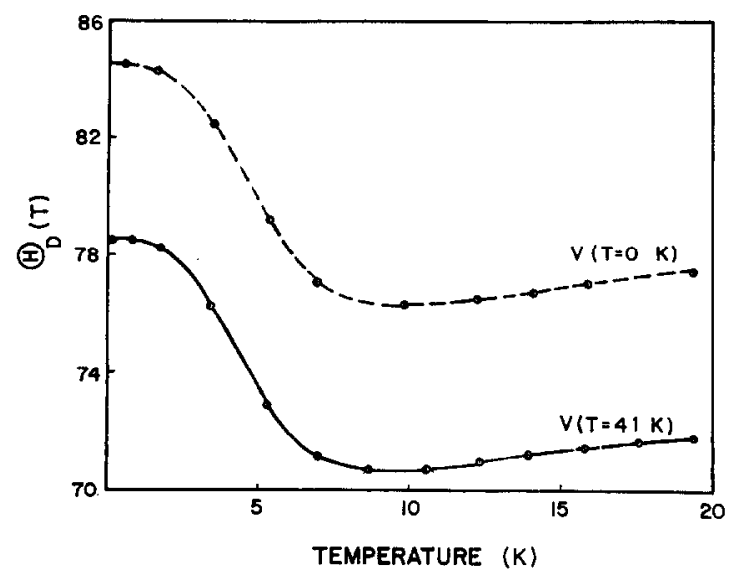

Fig. 7. Temperature-dependent Debye temperature $\Theta(T)$ tor $\Lambda \mathrm{r}$. Dashcd curve: calculated from $C_{V}[T, v(0 \mathrm{~K})]$; solid curve: calculated from $C_{V}[T, v(40 \mathrm{~K})]$.

thermal expansion curve for argon (fig. 5). For $\varepsilon$ and $\sigma$ the values appropriate for Ar were used (cf. sec. 3). The curve for $v(0 \mathrm{~K})$ was computed for a value of $\sigma / a=1.281$, the one for $v(40 \mathrm{~K})$ for a value of $\sigma / a=1.270$. The vibrational spectra at these densities are already rather different, which explains the difference between the two $C_{V}$ curves.

This difference is exhibited more clearly in fig. 7 where the temperaturedependent Debye temperature $\Theta(T)$ is plotted, calculated in two ways, namely from $C_{V}[T, v(0 \mathrm{~K})]$ and from $C_{V}[T, v(40 \mathrm{~K})]$, respectively. It will be obvious that if we had calculated $\Theta(T)$ from $C_{V}(T, p=0)$ we would have obtained a curve intermediate between the curves shown in fig. 7 , such that it coincides with the one at $T=0 \mathrm{~K}$ and with the other at $T=40 \mathrm{~K}$.

5. Mean-square amplitude of vibration. The mean-square amplitude of vibration is given by the expression

$$
\left\langle u^{2}\right\rangle=\frac{\hbar}{2 N M} \sum_{\boldsymbol{q}, j} \frac{\operatorname{coth}\left[\frac{1}{2} \beta \hbar \omega_{j}(\boldsymbol{q}, a)\right]}{\omega_{j}(\boldsymbol{q}, a)} .
$$

It is closely related to the Debye-Waller factor for scattering with momentum transfer $\boldsymbol{k}, v i z ., \exp [-2 W(\boldsymbol{k})]$. For cubic crystals $W(\boldsymbol{k})$ has the simple form

$$
W(\boldsymbol{k})=\frac{1}{6} k^{2}\left\langle u^{2}\right\rangle .
$$

In fig. 8 we have plotted the quantity $\left\langle u^{2}\right\rangle$ as a function of temperature. As in the case for the specific heat, $\left\langle u^{2}\right\rangle$ is shown for Ar for two fixed values of $v$, namely $v(0 \mathrm{~K})$ (dashed curve) and $v(40 \mathrm{~K})$ (solid curve). The function $\left\langle u^{2}(T)\right\rangle$, as it enters into the Debye-Waller factor, would follow a curve that coincides with the dashed one at $T=0 \mathrm{~K}$ and with the solid one at $40 \mathrm{~K}$. 


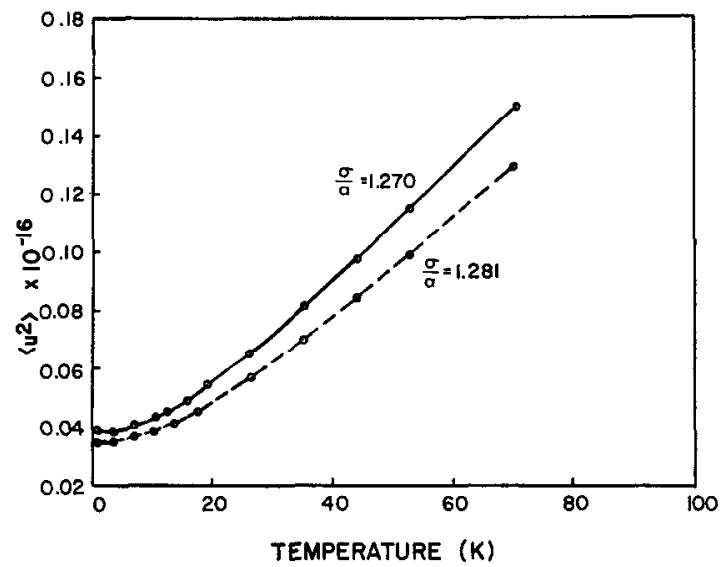

Fig. 8. Mean-square amplitude of vibration $\left\langle u^{2}\right\rangle$ as a function of temperature for Ar. Dashed curve: calculated with $a(0 \mathrm{~K})$; solid curve: calculated with $a(40 \mathrm{~K})$, both as determined from the minimization procedure.

For larger values of $T$ this curve would rise above the solid one. Experimental values of $2 W(T)$ are not available.

6. Summary. In this paper we have presented calculations of the thermal expansion, the specific heat, and the mean-square amplitude of vibration of a fcc crystal in which the particles interact through a Lennard-Jones pair potential. The calculations were carried out in the quasi-harmonic approximation; other approximations such as the use of high- or lowtemperature expansions have been avoided.

These calculations explicitly demonstrate the strong density dependence of the vibrational frequency distribution over the range of densities corresponding to those of the noble-gas solids. It turns out that the Grüneisen constant, although fairly constant for all the modes at a given density, is, itself, fairly strongly density dependent.

To illustrate the calculations we have chosen the case of argon. The potential constants for Ar were taken from a paper by Horton and Leech ${ }^{9}$ ). It appeared that these values of the potential parameters are not the optimal choice to fit the lattice constant a at $T=0 \mathrm{~K}$. They lead to a value $2 a(T=0)$ $=5.322 \AA$, whereas the experimental value is $5.300 \AA$, a difference of $0.4 \%$. A slight readjustment of the values of $\varepsilon$ and $\sigma$ could of course have led to the correct value of $2 a(T=0)$. However, whether this is worth the extra effort is questionable, particularly in view of demonstrated shortcomings of the Lennard-Jones potential ${ }^{13}$ ). At any rate, the quasi-harmonic approximation is shown to be able to reproduce experimental values of the coefficient of thermal expansion fairly well up to half the melting temperature. For 
higher temperatures anharmonic corrections would probably improve the theoretical results.

The curves for the specific heat and for the mean-square amplitude of vibration were calculated for the same values of the potential parameters. They were not meant to reproduce experimental values of these quantities for Ar quantitatively but they illustrate the volume dependence of these quantities as a consequence of the strong volume dependence of the vibrational spectrum.

Acknowledgement. One of the authors (F.W. de W.) wishes to thank the Netherlands Organization for Pure Research (Z.W.O.) for a visitors grant during the summer of 1969 which enabled him to complete this research.

\section{REFERENCES}

1) Nijboer, B. R. A. and de Wette, F. W., Physics Letters 17 (1965) 256.

2) See, for instance, Cowley, R. A., Rep. Prog. Phys. 31 (1968) 123.

3) For references we refer to Werthamer, N. R., Amer, J. Phys. 37 (1969) 763.

4) Pollack, G. L., Rev. mod. Phys. 36 (1964) 748.

5) Horton, G. K., Amer. J. Phys. 36 (1968) 93.

6) For $\sigma / a<1.2$ the eigenvalue problem shows some remarkable features. For a brief discussion see de Wette, F. W. and Nijboer, B. R. A., Phys. Letters 18 (1965) 19.

7) Misra, R. D., Proc. Cambridge Phil. Soc. 36 (1940) 173.

8) See e.g., C. Kittel, Introduction to Solid State Physics 3rd ed., John Wiley and Sons (New York, 1966) Chap. 6.

9) Horton, G. K. and Leech, J. W., Proc. Phys. Soc. 82 (1963) 816.

10) Peterson, O. G., Batchelder, D. N. and Simmons, R. O., Phys. Rev. 150 (1966) 703.

11) This confirms the results recently published by Leech, J. W. and Reissland, J. A., J. Phys. C: Solid St. Phys. 3 (1970) 975.

12) De Wette, F. W., Nosanow, L. H. and Werthamer, N. R., Phys. Rev. 162 (1967) 824.

13) Klein, M. I., Horton, G. K. and Feldman, J. L., Phys. Rev. 184 (1969) 968. 